\title{
Spin heat accumulation and spin-dependent temperatures in nanopillar spin valves
}

\author{
F. K. Dejene ${ }^{1 \star \dagger}$, J. Flipse ${ }^{1 \dagger}$, G. E. W. Bauer ${ }^{2,3}$ and B. J. van Wees ${ }^{1}$
}

\begin{abstract}
Since the discovery of the giant magnetoresistance effect ${ }^{1,2}$ the intrinsic angular momentum of the electron has opened up new spin-based device concepts. Our present understanding of the coupled transport of charge, spin and heat relies on the two-channel model for spin-up and spin-down electrons having equal temperatures. Here we report the observation of different (effective) temperatures for the spin-up and spindown electrons in a nanopillar spin valve subject to a heat current. By three-dimensional finite element modelling ${ }^{3}$ of our devices for varying thickness of the non-magnetic layer, spin heat accumulations (the difference of the spin temperatures) of $120 \mathrm{mK}$ and $350 \mathrm{mK}$ are extracted at room temperature and $77 \mathrm{~K}$, respectively, which is of the order of $10 \%$ of the total temperature bias over the nanopillar. This technique uniquely allows the study of inelastic spin scattering at low energies and elevated temperatures, which is not possible by spectroscopic methods.
\end{abstract}

Recent work in spin caloritronics ${ }^{4,5}$ aimed at spin-dependent thermoelectric effects led to the discovery of thermally driven spin sources ${ }^{6-10}$, cooling/heating by spin currents ${ }^{11,12}$, the magneto Seebeck ${ }^{13-16}$ and Seebeck rectification ${ }^{17}$ in magnetic tunnel junctions. Ref. 18 predicted spin-dependent temperatures in spin valve structures for sufficiently weak inter-spin heat exchange. The spin heat relaxation by inelastic scattering leads to a breakdown of the Wiedemann-Franz relation ${ }^{19}$ between the charge and electronic heat conductance of the spin valve ${ }^{18,20}$. Earlier experiments on the magnetic field dependence of the in-plane thermal conductance of magnetic multilayers ${ }^{21-24}$ focused on determining whether the giant magnetoresistance effect is dominated by either elastic or inelastic scattering. In these devices the change in heat resistance was found to be proportional to the (charge) magnetoresistance change because both are caused by (spin-dependent) interface scattering but no spin accumulations or spin-dependent temperatures build up.

A spin-dependent temperature builds up in spin valves when the thermal conductivity $(\kappa)$ in the ferromagnet $\left(\kappa_{\uparrow} \neq \kappa_{\downarrow}\right)$ is spin polarized and the spin flip and inelastic scattering are sufficiently weak $^{18,20}$. The Wiedemann-Franz relation tells us that the electronic part of the heat conductance in metals $\left(\kappa_{\mathrm{e}}\right)$ is proportional to the electrical conductivity $(\sigma)$, with a polarization $P_{\kappa}=\left(\kappa_{\mathrm{e} \uparrow}-\kappa_{\mathrm{e} \downarrow}\right) / \kappa_{\mathrm{e}}$ that should be equal to $P_{\sigma}=\left(\sigma_{\uparrow}-\sigma_{\downarrow}\right) / \sigma$. A heat current through a ferromagnetic metal ( $\mathrm{F}$ ) will therefore be spin polarized, creating a spin heat accumulation (SHA) by the spin-heat coupling at an interface with a non-magnetic metal (N; Fig. 1). If there would be no inelastic scattering of the electrons this SHA decays with the same spin relaxation length $\left(\lambda_{s}\right)$ as the spin accumulation, that is, the difference in the local chemical potential of the spin species. In real physical systems though, the ever-present inelastic phonon and electron-electron scattering leads to the exchange of heat between the two spin channels, thereby equilibrating the spin-up and spin-down temperatures $T_{\uparrow}$ and $T_{\downarrow}$ to the same average temperature (Fig. 1). Spin temperatures equilibrate over the spin heat relaxation length $\left(\lambda_{Q}\right)$, which at lower temperatures is limited by spin flip scattering $\left(\lambda_{Q}=\lambda_{\mathrm{s}}\right)$ and at high temperatures by inelastic scattering (when $\lambda_{Q}<\lambda_{\mathrm{s}}$ ). The thermal equivalent for the diffusion equation for the spin accumulation reads:

$$
\nabla^{2} T_{\mathrm{s}}=T_{\mathrm{s}} / \lambda_{Q}^{2}
$$

where $T_{\mathrm{s}}=T_{\uparrow}-T_{\downarrow}$ is the SHA. The temperature drop that builds up at the F/N interface (Fig. 1) then becomes (Supplementary Section SA):

$$
\Delta T=\frac{1}{2} P_{\kappa} T_{s}
$$

In regular current-perpendicular-to-plane spin valve devices inelastic scattering is caused by electron-phonon and electron-electron interactions $\mathrm{s}^{20}$. Time-domain thermoreflectance ${ }^{25}$ and ballisticelectron emission microscopy studies ${ }^{26}$ on inelastic scattering of hot electrons in copper found an inelastic (charge) equilibration length of the order of $60 \mathrm{~nm}$, which is more than five times smaller than $\lambda_{\mathrm{s}}=350 \mathrm{~nm}$ at room temperature ${ }^{27}$. As long as the copper

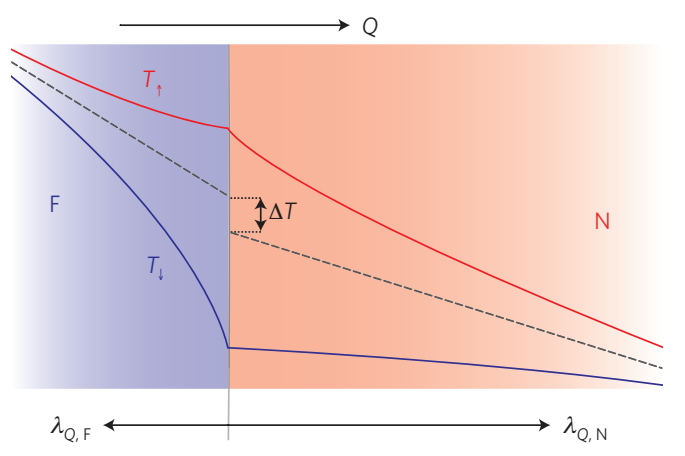

Figure 1 | SHA at an F/N interface. The spin polarized heat current in a ferromagnetic metal (F; blue shading) creates an SHA at the interface with a non-magnetic metal ( $\mathrm{N}$; red shading), because the heat currents have to be equally distributed over the spin channels in N. Inelastic scattering equilibrates the spin channel temperatures on the scale of the spin heat relaxation length $\lambda_{Q}$.

\footnotetext{
${ }^{1}$ Zernike Institute for Advanced Materials, Physics of Nanodevices, University of Groningen, 9747 AG Groningen, The Netherlands, ${ }^{2}$ Kavli Institute of NanoScience, Delft University of Technology, 2628 CJ Delft, The Netherlands, ${ }^{3}$ Institute for Materials Research and WPI Advanced Institute for Materials Research, Tohoku University, Sendai 980-8577, Japan. ${ }^{\dagger}$ These authors contributed equally to this work. *e-mail: F.K.Dejene@rug.nl
} 

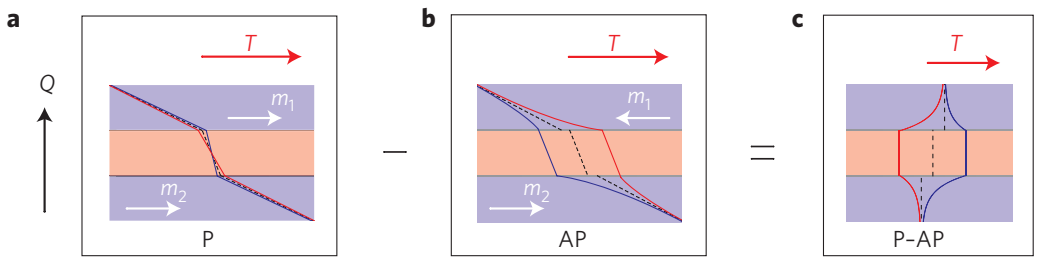

Figure 2 | SHA in an F/N/F spin valve. Temperature profiles over the stack in the $P$ and $A P$ configuration in the presence of a heat current (Q). $\mathbf{a}$, In the $P$ configuration the SHAs at both F/N interfaces have opposite signs, leading to a negligibly small SHA. $\mathbf{b}$, For the AP configuration the SHAs at the F/N interfaces have the same sign, creating a large SHA and a corresponding temperature drop between the F/N interfaces and the bulk of the F layers. c, A temperature drop between the P and AP configuration builds up owing to the spin heat valve effect.

spacer layer in a spin valve is comparable to $\lambda_{\mathrm{Q}}$ the SHA should be detectable by the second ferromagnetic layer. In Fig. 2, the spin-dependent temperatures $T_{\uparrow}$ and $T_{\downarrow}$ are plotted for the parallel (P) and antiparallel (AP) alignment of the magnetic layers in such a current-perpendicular-to-plane spin valve. For the $\mathrm{P}$ configuration the SHAs at both $\mathrm{F} / \mathrm{N}$ interfaces have opposite sign and sum up to be negligibly small. On the other hand, in the AP configuration both interfaces contribute constructively to generate a large SHA leading to a significant temperature drop $\Delta T$ (equation (1)) at both $\mathrm{F} / \mathrm{N}$ interfaces. If $\lambda_{Q}=\lambda_{\mathrm{s}}$ the Wiedemann-Franz relation holds, that is, the relative thermal conductance ratio $\left(\kappa_{\mathrm{P}}-\kappa_{\mathrm{AP}}\right) / \kappa_{\mathrm{p}}$ equals the giant magnetoresistance ratio $\left(\sigma_{\mathrm{P}}-\sigma_{\mathrm{AP}}\right) / \sigma_{\mathrm{p}}$ of the nanopillar. However, in the presence of inter-spin and spin-conserving inelastic scattering $\lambda_{\mathrm{Q}}<\lambda_{\mathrm{s}}$ and we may expect the Wiedemann-Franz relation to break down, because heat exchange short-circuits the spin channels, thereby decreasing $\kappa_{\mathrm{P}}-\kappa_{\mathrm{AP}}$ but not $\sigma_{\mathrm{P}}-\sigma_{\mathrm{AP}}$.

To observe the SHA, we use a nanopillar spin valve $\left(\mathrm{Ni}_{80} \mathrm{Fe}_{20} / \mathrm{Cu} / \mathrm{Ni}_{80} \mathrm{Fe}_{20}\right.$ stack with dimensions $150 \times 80 \mathrm{~nm}^{2}$ and a thickness of each layer of $15 \mathrm{~nm}$ ) as shown in Fig. 3. We measure the temperature of the bottom contact using a Pt-Constantan $\left(\mathrm{Ni}_{45} \mathrm{Cu}_{55}\right)$ thermocouple (contacts 3 and 4 ) while sending a charge current through the Pt-heater (contact 1 to 2). Both the thermocouple and the heater are electrically isolated from the bottom contact by an $\mathrm{Al}_{2} \mathrm{O}_{3}$ barrier $(\sim 8 \mathrm{~nm}$ thick). All samples were initially characterized by electrical measurements of the four-probe electrical resistance of the nanopillar using contacts 6 and 8 while sending a charge current from contact 5 to contact 7 . Using a standard lock-in technique ${ }^{9,12,28}$ with low excitation frequency (Methods), we separate the second harmonic voltage component $V^{2 f} \propto I^{2}$ from the first harmonic voltage response $V^{\text {lf }} \propto I$ (Methods). Measurements are carried out at room temperature as well as $77 \mathrm{~K}$.

To prove the existence of an SHA, we measure the thermovoltage $V^{2 f}$ by the $\mathrm{Pt}-\mathrm{Ni}_{45} \mathrm{Cu}_{55}$ thermocouple as a function of an inplane magnetic field, shown in Fig. 4a at room temperature. The second harmonic resistance $R^{2 f}=V^{2 f} / I^{2}$ is characterized by four abrupt changes corresponding to the switching from $\mathrm{P}$ to AP configurations and vice versa. On the right $y$ axis the difference between the thermocouple $\left(T_{\mathrm{TC}}\right)$ and reference temperature $\left(T_{0}=300 \mathrm{~K}\right)$ is plotted. The spin heat valve signal $R_{\mathrm{s}}^{2 \mathrm{f}}=R_{\mathrm{P}}^{2 \mathrm{f}}-R_{\mathrm{AP}}^{2 \mathrm{f}}$ of $-0.04 \mathrm{VA}^{-2}$ corresponds to a temperature difference of $-6 \mathrm{mK}$. At $77 \mathrm{~K}$ (Fig. $4 \mathrm{~b}$ ), the spin heat valve signal is $-0.06 \mathrm{~V} \mathrm{~A}^{-2}$, corresponding to a temperature change of $-17 \mathrm{mK}$ between $\mathrm{P}$ and AP configurations. The background resistance, $R_{\mathrm{b}}^{2 \mathrm{f}}=\left(R_{\mathrm{P}}^{2 \mathrm{f}}+R_{\mathrm{AP}}^{2 \mathrm{f}}\right) / 2$, is lower at $77 \mathrm{~K}\left(21.15 \mathrm{~V} \mathrm{~A}^{-2}\right)$ than at room temperature $\left(29.13 \mathrm{~V} \mathrm{~A}^{-2}\right)$ owing to the reduced resistance of the heater. Similar values are found for two other samples from the same batch (Supplementary Section SB).

In Fig. 4c we show the four-probe electrical resistance of the nanopillar at room temperature as a function of the external magnetic field measured using contacts 6 and 8 while a charge current flows from contact 5 to contact 7. A spin valve signal of $-80 \mathrm{~m} \Omega$ is observed on a background resistance of $2.27 \Omega$. By using the three-dimensional finite element model (3D-FEM) to fit the spin valve signals, we obtain a spin polarization $\mathrm{P}_{\sigma}$ of 0.52 , typical of the bulk spin polarization for Permalloy ${ }^{12,28,29}$. As a consistency check, the spin-dependent Seebeck ${ }^{9,28}$ and Peltier effects ${ }^{12}$ are also measured in the same device (see Supplementary Section SC).

Fitting the measured spin heat valve signal of $-0.04 \mathrm{~V} \mathrm{~A}^{-2}$ to the spin heat diffusion model under the assumption of equal polarizations $P_{\kappa}$ and $P_{\sigma}$ (Supplementary Sections SA and SE) leads to a spin heat relaxation length $\lambda_{Q, P y}$ of $1 \mathrm{~nm}$ in Permalloy, which is one-fifth of its spin relaxation length of $5 \mathrm{~nm}$ (ref. 30). Taking the same scaling for the copper layer we obtain a $\lambda_{Q, \mathrm{Cu}}$ of $70 \mathrm{~nm}$ as one-fifth of $\lambda_{\mathrm{s}, \mathrm{Cu}}=350 \mathrm{~nm}$ (ref. 27). In another set of samples, we measured the SHA for varying thickness of the Cu layer $\left(t_{N}=5,15\right.$ and $\left.60 \mathrm{~nm}\right)$. This allows us to obtain $\lambda_{\mathrm{Q}, \mathrm{Cu}}$ of $45 \mathrm{~nm}$ in close agreement with the value obtained above (Supplementary Section SI). The fact that we
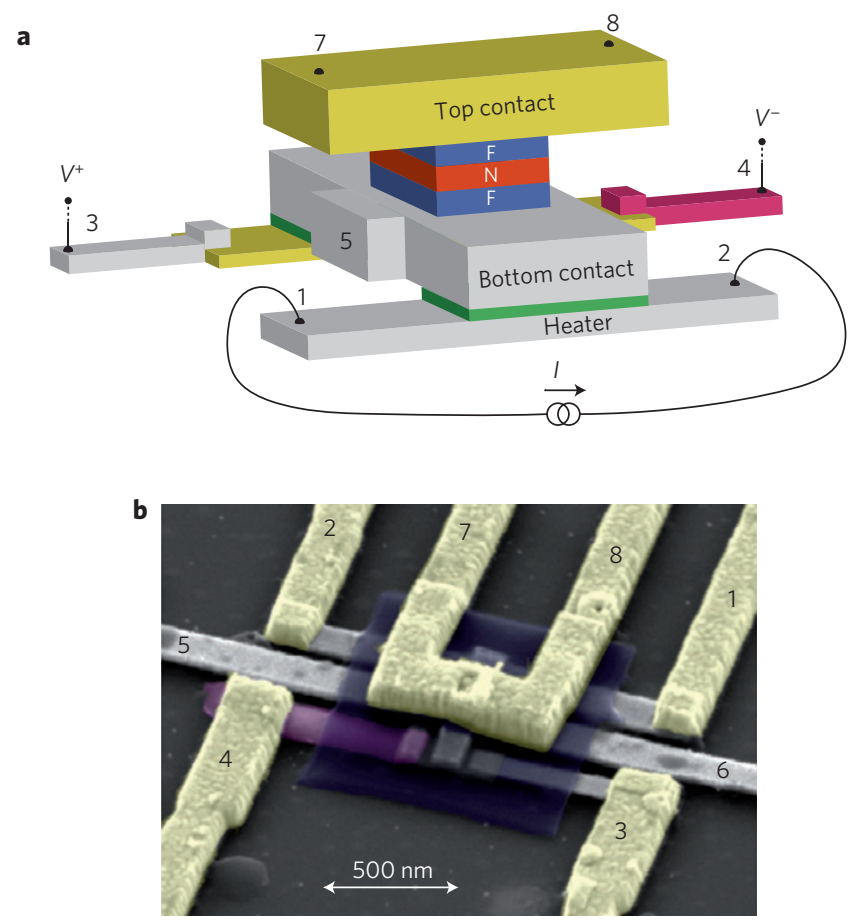

Figure 3 | Device geometry. a, Schematics of the measured device showing an $\mathrm{F} / \mathrm{N} / \mathrm{F}$ pillar spin valve sandwiched between Au top and Pt bottom contacts. A charge current $/$ through the Pt-heater (contact 1 and 2 ) increases the temperature of the bottom contact, which is simultaneously measured by a Pt-Constantan $\left(\mathrm{Ni}_{45} \mathrm{Cu}_{55}\right)$ thermocouple. Both the heater and thermocouple are electrically isolated from the bottom contact by an $\mathrm{Al}_{2} \mathrm{O}_{3}$ barrier (green; $8 \mathrm{~nm}$ thick) to avoid any charge-related spurious signals. b. Coloured 3D scanning electron microscope image of the measured device. The nanopillar sits half way between the Pt-Ni ${ }_{45} \mathrm{Cu}_{55}$ thermocouple (contacts 3 and 4 ) and the Pt-Joule heater (contacts 1 and 2). Crosslinked polymethyl methacrylate (blue) electrically isolates the bottom contact (grey contacts 5 and 6) from the top contact (contacts 7 and 8). 

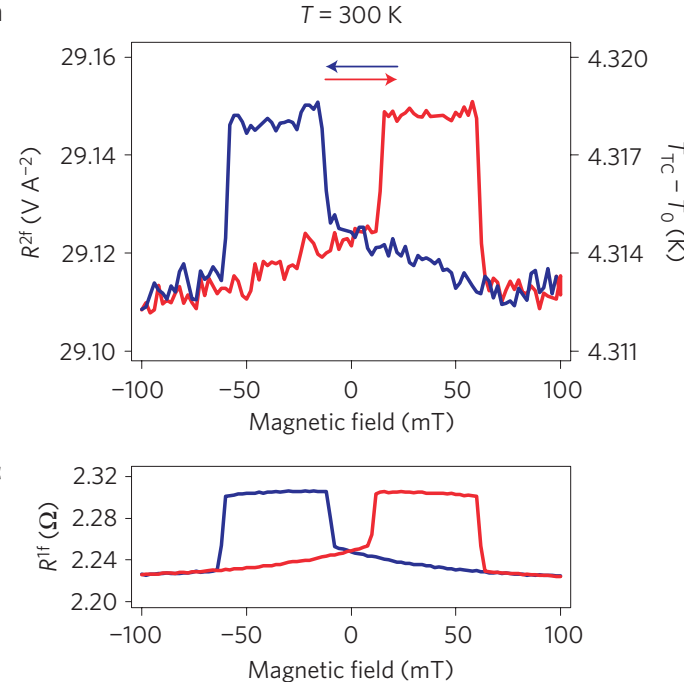

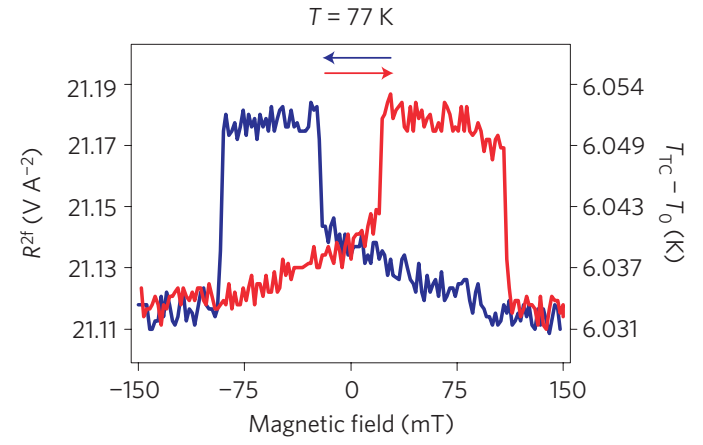

d

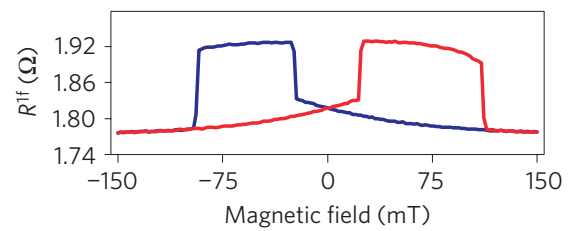

Figure 4 | Measured spin heat and conventional spin valve effects. a,b, Second harmonic response $R^{2 f}=V^{2 f} / I^{2}$ measured at the thermocouple, at room temperature (a) and at $77 \mathrm{~K}(\mathbf{b})$, both for an r.m.s. current of $2 \mathrm{~mA}$ through the heater. Red and blue curves show forward ( $-\mathbf{H} \rightarrow \mathbf{H}$ ) and backward $(\mathbf{H} \rightarrow-\mathbf{H})$ traces of the applied magnetic field. On the right axes the second harmonic r.m.s. value of the temperature differences are shown at the

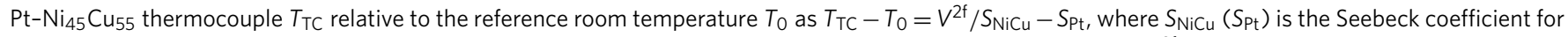
$\mathrm{Ni}_{45} \mathrm{Cu}_{55}\left(\mathrm{Pt}\right.$ ) (Supplementary Table $\mathrm{SI}$ ) and $T_{0}$ is taken as $300 \mathrm{~K}$ in $\mathbf{a}$ and $77 \mathrm{~K}$ in $\mathbf{~} \mathbf{b}$. The heat resistance $R_{Q \text {,pillar }} \propto R^{2 f}$ of the nanopillar and therefore the temperature is larger in the AP than the P configuration. c,d, Four-probe electrical resistances $R^{1 \mathrm{ff}}=V^{1 \mathrm{ff}} / /$ as a function of magnetic field measured using contacts 6 and 8 while current flows from contact 5 to contact 7 for room temperature (c) and $77 \mathrm{~K}$ (d).

observe SHA up to $60 \mathrm{~nm}$ and that $\lambda_{\mathrm{Q}, C u}>t_{\mathrm{N}}$ shows that inter-spin and electron-phonon inelastic scattering is surprisingly weak in nanopillar devices even at room temperature.

Most material-dependent transport parameters at $77 \mathrm{~K}$ can be found in the literature (Supplementary Table S1). To fit the measured spin valve signal at $77 \mathrm{~K}$ of $-160 \mathrm{~m} \Omega$ (Fig. 4d), we require a slightly higher spin polarization $P_{\sigma}$ of 0.59 , in agreement with earlier reports ${ }^{25}$. From the measured spin heat valve signal of $-0.06 \mathrm{~V} \mathrm{~A}^{-2}$ and $P_{\kappa}=P_{\sigma}=0.59$, we obtain a $\lambda_{Q, \mathrm{Cu}}$ of $150 \mathrm{~nm}$, more than two times longer than the $\lambda_{Q, \mathrm{Cu}}$ at room temperature, demonstrating the reduced inelastic scattering.

From the 3D-FEM and the above experimental results we can now estimate the difference in the effective temperatures of the spin-up and the spin-down channels in the copper layer. We find $T_{\uparrow}-T_{\downarrow}=120 \mathrm{mK}$ (at room temperature) and $350 \mathrm{mK}$ (at $77 \mathrm{~K}$ ), up to $10 \%$ of the temperature bias of $4 \mathrm{~K}$ across the nanopillar for a root-mean-square (r.m.s.) current of $2 \mathrm{~mA}$ through the heater. Here, the temperature bias is defined as the temperature difference between the bottom Pt/Py and the top Py/Au interface. In addition to the vertical temperature difference over the nanopillar a horizontal temperature gradient exists, parallel to the $\mathrm{Py} / \mathrm{Cu}$ interface. The horizontal temperature gradient is only $6 \%$ of the vertical temperature gradient (Supplementary Section SF) and does not lead to any SHA. In our modelling we do not take into account electrical or heat interface resistances ${ }^{30}$. We would like to emphasize that those would not modify the extracted values of the SHA (Supplementary Section SH).

The SHA is a unique concept that deserves more study. Our results indicate that the spin heat relaxation length in copper is close to the recently measured charge heat relaxation length ${ }^{25,26}$. Indeed, at higher temperatures the inelastic scattering is thought to be dominated by phonons and is not spin selective. We should therefore interpret the results not as a temperature difference of thermalized spin channels. The SHA is rather a measure of the difference between non-thermalized spin distributions that can be parameterized by the effective temperature parameter ${ }^{20}$.

We measured the difference between the effective temperatures for spin-up and spin-down electrons in heat current-biased nanopillar spin valves. Modulating the heat conductance of the nanopillar by the magnetization configurations allows control of the flow of heat across the nanopillar, opening up possibilities for room-temperature magnetic thermal switches. Whereas optical pump and probe techniques and hot-electron transistors can access spin-dependent relaxation processes only at high energies, conventional transport experiments are limited to very low temperatures. The spin heat valve measurement, on the contrary, offers a unique possibility to estimate inelastic scattering lengths at the Fermi energy both at low and elevated temperatures.

\section{Methods}

Fabrication. One optical lithography step followed by eleven electron-beam lithography steps were employed to make the device. For each step, materials were electron-beam evaporated except for the $\mathrm{Ni}_{45} \mathrm{Cu}_{55}$ alloy, which was sputtered to maintain the bulk stoichiometry. First, a 40-nm-thick Pt Joule heater was deposited on a thermally oxidized $\mathrm{Si}$ substrate. Then, the Pt-Constantan $\left(\mathrm{Ni}_{45} \mathrm{Cu}_{55}\right)$ thermocouple was realized on top of a 10-nm-thick Au layer. This is followed by a deposition of an 8-nm-thick $\mathrm{Al}_{2} \mathrm{O}_{3}$ layer over the Pt-Joule heater and the thermocouple to electrically isolate them from the bottom contact of the nanopillar. The insulating layer prevents the pick-up of any charge-related effects. A Pt bottom contact $(60 \mathrm{~nm}$ thick) is then deposited on top of the heater and thermocouple. In the next step, $\mathrm{Ni}_{80} \mathrm{Fe}_{20}(15) / \mathrm{Cu}\left(\mathrm{t}_{\mathrm{Cu}}=5,15\right.$ and 60$) / \mathrm{Ni}_{80} \mathrm{Fe}_{20}$ (15)/Au(10), where the numbers in parentheses are the thicknesses in nanometres, was deposited without breaking the vacuum of the deposition chamber. Crosslinked polymethyl methacrylate around the nanopillar prevents short-circuiting between the bottom and the top contact (130-nm-thick Au).

Measurements and modelling. All measurements were done using a standard lock-in technique at low frequency $(f<20 \mathrm{~Hz})$ such that a quasi-steady-state condition is reached and at the same time capacitive and inductive coupling are suppressed. As a measured signal $V$ has both linear and nonlinear contributions given as $V=I R^{1 \mathrm{f}}+I^{2} R^{2 \mathrm{f}}$, we used a multiple lock-in measurement to distinguish the first harmonic resistance $R^{1 \mathrm{f}}=V^{1 \mathrm{f}} / I$ from the second harmonic resistance $R^{2 \mathrm{f}}=V^{2 \mathrm{f}} / I^{2}$. To fully characterize the samples, four different measurements were performed. First, in the spin valve measurements, the four-probe resistance of the nanopillar was measured as a function of magnetic field from which the bulk conductivity polarization $\left(P_{\sigma}\right)$ was obtained. Then we measure the spin-dependent Seebeck and spin-dependent Peltier effect in the same device. From these measurements, the spin polarizations of the Seebeck $\left(P_{\mathrm{S}}\right)$ and Peltier coefficients $\left(P_{\Pi}\right)$ are obtained. By using the 3D-FEM (Supplementary Section SE) together with the extracted values for $P_{\sigma}, P_{\mathrm{S}}$ and $P_{\Pi}$, we determine the spin heat relaxation length. Measurements were taken both at room temperature and $77 \mathrm{~K}$. 
Received 11 January 2013; accepted 5 August 2013; published online 8 September 2013

\section{References}

1. Baibich, M. N. et al. Giant magnetoresistance of (001)Fe/(001)Cr magnetic superlattices. Phys. Rev. Lett. 61, 2472-2475 (1988).

2. Binasch, G., Grünberg, P., Saurenbach, F. \& Zinn, W. Enhanced magnetoresistance in layered magnetic structures with antiferromagnetic interlayer exchange. Phys. Rev. B 39, 4828-4830 (1989).

3. Slachter, A., Bakker, F. L. \& van Wees, B. J. Modeling of thermal spin transport and spin-orbit effects in ferromagnetic/nonmagnetic mesoscopic devices. Phys. Rev. B 84, 174408 (2011).

4. Bauer, G. E.W., MacDonald, A. H. \& Maekawa, S. Spin caloritronics. Solid State Commun. 150, 459-460 (2010).

5. Bauer, G. E. W., Saitoh, E. \& van Wees, B. J. Spin caloritronics. Nature Mater. 11,391-399 (2012).

6. Uchida, K. et al. Observation of the spin Seebeck effect. Nature 455, 778-781 (2008).

7. Jaworski, C. M. et al. Observation of the spin-Seebeck effect in a ferromagnetic semiconductor. Nature Mater. 9, 898-903 (2010).

8. Uchida, K. et al. Spin Seebeck insulator. Nature Mater. 9, 894-897 (2010).

9. Slachter, A., Bakker, F. L., Adam, J-P. \& van Wees, B. J. Thermally driven spin injection from a ferromagnet into a non-magnetic metal. Nature Phys. 6, 879-882 (2010).

10. Le Breton, J-C., Sharma, S., Saito, H., Yuasa, S. \& Jansen, R. Thermal spin current from a ferromagnet to silicon by Seebeck spin tunneling. Nature 475, 82-85 (2011).

11. Gravier, L., Serrano-Guisan, S., Reuse, F. \& Ansermet, J-Ph. Spin-dependent Peltier effect of perpendicular currents in multilayered nanowires. Phys. Rev. B 73, 052410 (2006).

12. Flipse, J., Bakker, F. L., Slachter, A., Dejene, F. K. \& van Wees, B. J. Direct observation of the spin-dependent Peltier effect. Nature Nanotech. 7, 166-168 (2012).

13. Walter, M. et al. Seebeck effect in magnetic tunnel junctions. Nature Mater. 10, 742-746 (2011).

14. Liebing, N. et al. Tunneling magnetothermopower in magnetic tunnel junction nanopillars. Phys. Rev. Lett. 107, 177201 (2011).

15. Lin, W. et al. Giant spin-dependent thermoelectric effect in magnetic tunnel junctions. Nature Commun. 3, 744 (2012).

16. Czerner, M., Bachmann, M. \& Heiliger, C. Spin caloritronics in magnetic tunnel junctions: Ab initio studies. Phys. Rev. B 83, 132405 (2011).

17. Zhang, Z. H. et al. Seebeck rectification enabled by intrinsic thermoelectrical coupling in magnetic tunneling junctions. Phys. Rev. Lett. 109, 037206 (2012).

18. Hatami, M., Bauer, G. E. W., Zhang, Q. \& Kelly, P. J. Thermal spin-transfer torque in magnetoelectronic devices. Phys. Rev. Lett. 99, 066603 (2007).

19. Franz, R. \& Wiedemann, G. Ueber die Wärme-Leitungsfähigkeit der Metalle. Ann. Phys. 165, 497-531 (1853).

20. Heikkilä, T. T., Hatami, M. \& Bauer, G. E. W. Spin heat accumulation and its relaxation in spin valves. Phys. Rev. B 81, 100408 (2010).
21. Sato, H., Aoki, Y., Kobayashi, Y., Yamamoto, H. \& Shinjo, T. Giant magnetic field effect on thermal conductivity of magnetic multilayers, $\mathrm{Cu} / \mathrm{Co} / \mathrm{Cu} / \mathrm{Ni}(\mathrm{Fe})$. J. Phys. Soc. Jpn 62, 431-434 (1993).

22. Sato, H., Aoki, Y., Kobayashi, Y., Yamamoto, H. \& Shinjo, T. Huge magnetic field-dependent thermal conductivity in magnetic multilayer films. J. Magn. Magn. Mater. 126, 410-412 (1993).

23. Jeong, T., Moneck, M. T. \& Zhu, J-G. Giant magneto-thermal conductivity in magnetic multilayers. IEEE Trans. Magn. 48, 3031-3034 (2012).

24. Kimling, J., Nielsch, K., Rott, K. \& Reiss, G. Field-dependent thermal conductivity and Lorenz number in Co/Cu multilayers. Phys. Rev. B 87, 134406 (2013).

25. Wang, W. \& Cahill, D. G. Limits to thermal transport in nanoscale metal bilayers due to weak electron-phonon coupling in Au and Cu. Phys. Rev. Lett. 109, 175503 (2012).

26. Parui, S., van der Ploeg, J. R. R., Rana, K. G. \& Banerjee, T. Nanoscale hot electron transport across $\mathrm{Cu} / \mathrm{n}-\mathrm{Si}(100)$ and $\mathrm{Cu} / \mathrm{n}-\mathrm{Si}(111)$ interfaces. Phys. Status Solidi 5, 388-390 (2011).

27. Jedema, F. J., Filip, A. T. \& Wees, B. J. van Electrical spin injection and accumulation at room temperature in an all-metal mesoscopic spin valve. Nature 410, 345-348 (2001).

28. Dejene, F. K., Flipse, J. \& Van Wees, B. J. Spin-dependent Seebeck coefficients of $\mathrm{Ni}_{80} \mathrm{Fe}_{20}$ and $\mathrm{Co}$ in nanopillar spin valves. Phys. Rev. B 86, 024436 (2012).

29. Zhu, M., Dennis, C. L. \& McMichael, R. D. Temperature dependence of magnetization drift velocity and current polarization in $\mathrm{Ni}_{80} \mathrm{Fe}_{20}$ by spin-wave Doppler measurements. Phys. Rev. B 81, 140407 (2010).

30. Bass, J. \& Pratt, W. P. Spin-diffusion lengths in metals and alloys, and spin-flipping at metal/metal interfaces: an experimentalist's critical review. J. Phys. Condens. Matter 19, 183201 (2007).

\section{Acknowledgements}

We would like to acknowledge B. Wolfs, M. de Roosz and J. G. Holstein for technical assistance. This work is part of the research programme of the Foundation for Fundamental Research on Matter (FOM) and supported by NanoLab NL, EU FP7 ICT Grant No. 251759 MACALO, JSPS Grand-in-Aid for Scientific Research A No. 25247056, Deutsche Forschungsgemeinschaft (DFG) Priority Programme SPP 1538 'Spin-Caloric Transport' and the Zernike Institute for Advanced Materials.

\section{Author contributions}

F.K.D., J.F. and B.J.v.W. conceived the experiments. F.K.D. and J.F. designed and carried out the main experiments. All authors were involved in the analysis. F.K.D. and J.F. wrote the paper, with the help of the co-authors.

\section{Additional information}

Supplementary information is available in the online version of the paper. Reprints and permissions information is available online at www.nature.com/reprints. Correspondence and requests for materials should be addressed to F.K.D.

\section{Competing financial interests}

The authors declare no competing financial interests. 